\title{
Signatures of Transverse Magnetic Anisotropy in Transport through a Large-Spin Molecule in the Kondo Regime
}

\author{
M. MISIORNY ${ }^{a, *}$, I. WEYMANN ${ }^{a}$ AND J. BARNAS $\hat{S}^{a, b}$ \\ ${ }^{a}$ Faculty of Physics, Adam Mickiewicz University, 61-614 Poznań, Poland \\ ${ }^{b}$ Institute of Molecular Physics, Polish Academy of Sciences, 60-179 Poznań, Poland
}

\begin{abstract}
The effect of transverse magnetic anisotropy on spin-dependent transport through a large-spin molecule strongly tunnel-coupled to ferromagnetic electrodes is analyzed theoretically. In particular, we investigate whether it is possible to observe in transport signatures of oscillations of the ground-state doublet splitting due to the application of an external magnetic field along the molecule's hard axis. We show that magnetic field leads to revivals of the Kondo effect, with the Kondo temperature depending on the magnetic configuration of the device.
\end{abstract}

DOI: $10.12693 /$ APhysPolA.128.200

PACS: 72.10.Fk, 72.25.-b, 73.23.-b, 75.50.Xx

\section{Introduction}

The interest in spin-dependent transport through large-spin nanoscopic systems, such as single-molecule magnets [1] or atoms [2], is driven by the prospect of encoding a bit of information into the physical state of the system's spin being "up" or "down" [3]. The prerequisite for the required bistability is the uniaxial spinanisotropy of an "easy-axis" type, so that the two opposite spin states along a specific axis are energetically favored. The key obstacle for achieving this bistability is, however, the transverse anisotropy that generates spintunneling between the "up" and "down" states [4-7], providing thus a channel for information loss. For this reason, the knowledge of how the transverse spin-anisotropy affects transport properties of a nanoscopic system is of vital importance.

Here, we focus on analyzing the spin-dependent transport through a model magnetic molecule strongly tunnelcoupled to ferromagnetic electrodes. Since in such a case spin-exchange (Kondo) correlations are in action, one can expect anomalous signatures in transport to emerge at low temperatures [8]. For molecules characterized by the spin number larger than $1 / 2$, the magnetic anisotropy comes into picture and determines whether the Kondo effect occurs or not. In a bistable system, the uniaxial component of magnetic anisotropy generally suppresses the effect [9-12], whereas the presence of an additional transverse component leads to its restoration $[13,14]$. In particular, in this work we consider the possibility of observing the effect of transverse magnetic anisotropy on molecule's transport characteristics in the presence of external magnetic field applied along the molecule's hard axis.

* corresponding author; e-mail: misiorny@amu.edu.pl

\section{Theoretical description}

Magnetic molecules can be described by a model consisting of a magnetic core of spin $S>1 / 2$, which is exchange coupled with strength $J>0$ to a single conducting orbital level (OL). The respective Hamiltonian has the form

$$
\hat{\mathcal{H}}_{\mathrm{mol}}=\hat{\mathcal{H}}_{\mathrm{OL}}+\hat{\mathcal{H}}_{\mathrm{S}}-J \hat{\boldsymbol{s}} \cdot \hat{\boldsymbol{S}}+B_{x}\left(\hat{s}_{x}+\hat{S}_{x}\right)
$$

The orbital level is described by $\hat{\mathcal{H}}_{\mathrm{OL}}=\varepsilon \sum_{\sigma} \hat{n}_{\sigma}+U \hat{n}_{\uparrow} \hat{n}_{\downarrow}$, with $\hat{n}_{\sigma}=\hat{c}_{\sigma}^{\dagger} \hat{c}_{\sigma}$ and $\hat{c}_{\sigma}^{\dagger}$ standing for the creation operator for a spin- $\sigma$ electron of energy $\varepsilon$ in the OL. The Coulomb energy of two electrons of opposite spins in the orbital is accounted by $U$. We assume that only the core spin is subject to magnetic anisotropy, and its magnetic properties are represented by the giant-spin Hamiltonian [1]:

$$
\hat{\mathcal{H}}_{\mathrm{S}}=-D \hat{S}_{z}^{2}+(E / 2)\left(\hat{S}_{+}^{2}+\hat{S}_{-}^{2}\right) .
$$

Here, $D$ and $E$ are the uniaxial and transverse anisotropy constants, respectively, and $\hat{S}_{ \pm}=\hat{S}_{x} \pm i \hat{S}_{y}$ with $\hat{S}_{j}(j=$ $x, y, z)$ being the $j$-th component of the molecule's core spin operator $\hat{\boldsymbol{S}}$. Moreover, we focus on the case of magnetic anisotropy of an easy axis type $(D>0)$, and assume $E>0$, which varies within the range $0 \leqslant E / D \leqslant 1 / 3$. The next term of $\hat{\mathcal{H}}_{\mathrm{mol}}$ is responsible for the ferromagnetic $(J>0)$ exchange interaction between the spin $\hat{\boldsymbol{S}}$ of the molecule's magnetic core and the spin $s$ of an electron residing in the OL, with $\hat{\boldsymbol{s}}=\frac{1}{2} \sum_{\sigma \sigma^{\prime}} \hat{c}_{\sigma}^{\dagger} \hat{\boldsymbol{\sigma}}_{\sigma \sigma^{\prime}} \hat{c}_{\sigma^{\prime}}$ and $\hat{\boldsymbol{\sigma}} \equiv\left(\hat{\sigma}^{x}, \hat{\sigma}^{y}, \hat{\sigma}^{z}\right)$ denoting the Pauli spin operator. Finally, the last term of Eq. (1) accounts for the Zeeman interaction, where $B_{x}$ corresponds to an external magnetic field (measured in energy units, i.e. $g \mu_{\mathrm{B}} \equiv 1$ ) applied along the molecule's hard axis.

Transport of electrons through the system takes place only via the OL, which is tunnel-coupled to two ferromagnetic metallic electrodes. The $q$-th electrode $[q=$ (L)eft, (R)ight] is modelled as a reservoir of noninteracting itinerant electrons, and described by $\hat{\mathcal{H}}_{\mathrm{el}}^{q}=$ $\sum_{\sigma} \int_{-W}^{W} \mathrm{~d} \epsilon \epsilon \hat{a}_{q \sigma}^{\dagger}(\epsilon) \hat{a}_{q \sigma}(\epsilon)$, where $\hat{a}_{q \sigma}^{\dagger}(\epsilon)$ is the creation operator of a spin- $\sigma$ electron and $W$ denotes the band half-width, taken as energy unit $W \equiv 1$. We restrict 
the discussion to the situation when magnetic moments of electrodes are collinear (parallel or antiparallel), and their orientation also coincides with that of the system's easy axis. In such a case, tunneling of electrons between the molecule and electrodes is characterized by

$$
\hat{\mathcal{H}}_{\text {tun }}=\sum_{q \sigma} \sqrt{\frac{\Gamma_{\sigma}^{q}}{\pi}} \int_{-W}^{W} \mathrm{~d} \epsilon\left[\hat{a}_{q \sigma}^{\dagger}(\epsilon) \hat{c}_{\sigma}+\hat{c}_{\sigma}^{\dagger} \hat{a}_{q \sigma}(\epsilon)\right],
$$

with $\Gamma_{\sigma}^{q}$ representing the strength of spin-dependent tunnel coupling between the OL and the $q$-th electrode. If both electrodes are made of the same material, described by the spin polarization coefficient $P$, the hybridization for majority ("+")/minority ("-") electrons in the $q$-th electrode can be parameterized as $\Gamma_{ \pm}^{q}=$ $(\Gamma / 2)(1 \pm P)$.

At low temperature $T$ and low bias voltage $V_{\mathrm{b}}$, the differential conductance of the system can be approximated by the symmetrized equilibrium spectral function $[15,16]$ :

$$
\frac{\mathrm{d} I}{\mathrm{~d} V_{\mathrm{b}}} \stackrel{T \rightarrow 0}{\approx} \frac{2 e^{2}}{h} \pi \sum_{\sigma} \frac{2 \Gamma_{\sigma}^{\mathrm{L}} \Gamma_{\sigma}^{\mathrm{R}}}{\Gamma_{\sigma}^{\mathrm{L}}+\Gamma_{\sigma}^{\mathrm{R}}} \frac{A_{\sigma}\left(\frac{e V_{\mathrm{b}}}{2}\right)+A_{\sigma}\left(-\frac{e V_{\mathrm{b}}}{2}\right)}{2}
$$

with the spin-dependent spectral function of the OL given by $A_{\sigma}(\omega)=-(1 / \pi) \operatorname{Im}\left\langle\left\langle\hat{c}_{\sigma} \mid \hat{c}_{\sigma}^{\dagger}\right\rangle\right\rangle_{\omega}^{\mathrm{r}}$, where $\left\langle\left\langle\hat{c}_{\sigma} \mid \hat{c}_{\sigma}^{\dagger}\right\rangle\right\rangle_{\omega}^{\mathrm{r}}$ is the Fourier transform of the retarded Green function of the OL. The spectral function $A_{\sigma}(\omega)$ is calculated by means of the Wilson numerical renormalization group (NRG) method [17, 18] with a full density matrix [19]. Here, in particular, we employ the Budapest Flexible DM-NRG code [20]. In NRG procedure the conduction band is discretized logarithmically with a discretization parameter $\Lambda$ and the whole problem is solved in an iterative fashion by retaining $N_{k}$ states during iteration. For the present problem we exploited the Abelian symmetry for the total charge and used $\Lambda=1.8$ and $N_{k}=2500$.

\section{Results and discussion}

It has already been demonstrated that if a molecule is bistable, i.e., characterized exclusively by uniaxial magnetic anisotropy $(D>0$ and $E=0)$, the Kondo effect is generally suppressed, which is especially visible in the antiparallel magnetic configuration of electrodes [11, 12]. On the other hand, in the parallel configuration due to the presence of an effective exchange field [21-23] the influence of magnetic anisotropy is limited only to the particle-hole symmetry point $(\varepsilon=-U / 2)$ where the field disappears. The reason behind such a behavior is that the spin-exchange processes between the two metastable ground states responsible for the formation of the Kondo resonance are not allowed. Interestingly enough, when the transverse component of magnetic anisotropy is included $(E \neq 0)$ the situation changes dramatically, as this component [see the second term of Eq. (2)] makes possible mixing of the core spin states, and thus, the spin-exchange processes become active again. Specifically, in such a case, a molecule with a half-integer spin $S^{t}=S+s$ effectively behaves as a one-a-half pseudospin, and one can observe the revival of the Kondo effect [14].

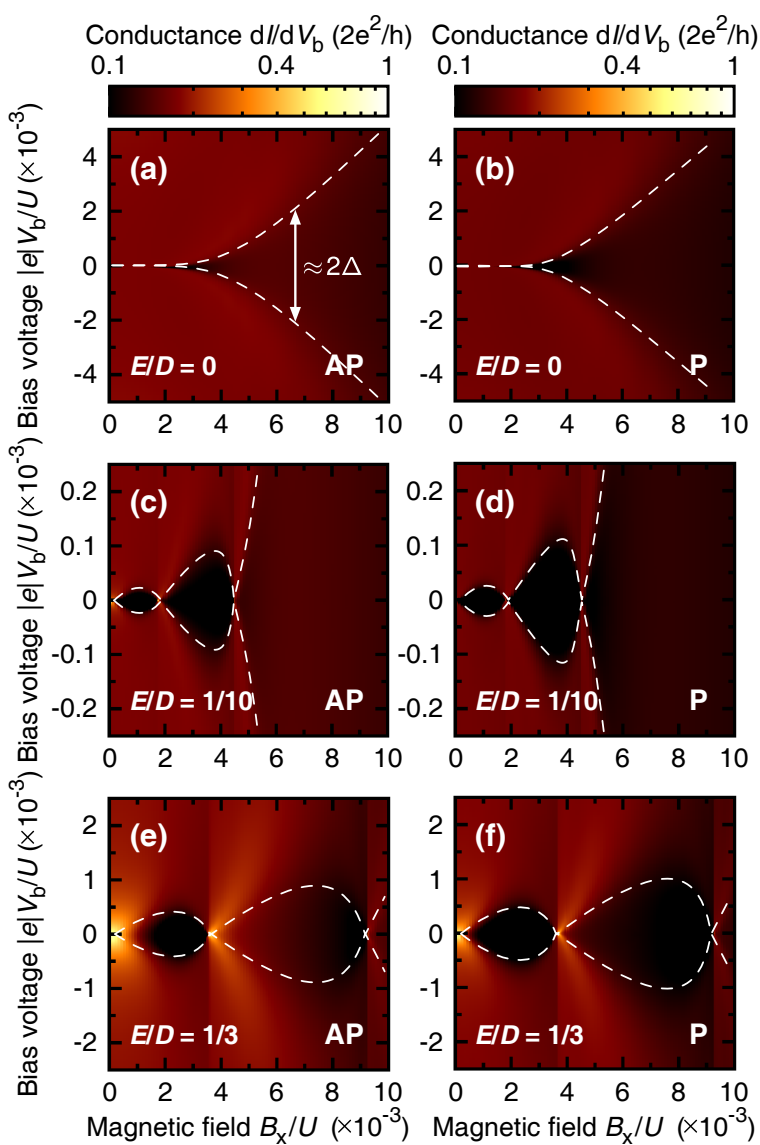

Fig. 1. Differential conductance as a function of magnetic field $B_{x}$ applied along the molecule's hard axis and bias voltage $V_{\mathrm{b}}$ for indicated values of $E / D$. Left/Right column corresponds to the antiparallel/parallel magnetic configuration of the leads. Dashed lines serve as a guide for eyes and schematically depict excitation energy within the ground state doublet. Parameters: $S=2, U / W=0.4$ (with $W \equiv 1$ being the energy unit), $\varepsilon=-U / 2, J / U=0.01125, D / U=3.75 \times 10^{-3}$, $\Gamma / U=0.1, P=0.5$ and $T=0$.

A feature characteristic to nanoscopic large-spin systems exhibiting both uniaxial and transverse magnetic anisotropy are oscillations of the tunnel splitting $\Delta$ of the ground state when an external magnetic field is applied along the system's hard anisotropy axis [1,24]. Since at low temperatures transport properties of the system are fully determined by its ground state doublet, it is thus interesting to analyze how the oscillations of the ground state splitting affect respective transport characteristics. In general, at low bias voltage one then expects to observe excitations within the ground state doublet. For this purpose, in Fig. 1 we present the density plot of the differential conductance $\mathrm{d} I / \mathrm{d} V_{\mathrm{b}}$ as a function of an external magnetic field $B_{x}$ applied along the hard $(x)$ axis and the bias voltage $V_{\mathrm{b}}$ calculated for $\varepsilon=-U / 2$.

Let us first consider the case when transverse magnetic anisotropy is absent $(E=0)$, Fig. 1a,b. As mentioned above, for $B_{x}=0$ the ground state doublet is degenerate 
and the Kondo effect is suppressed due to the uniaxial magnetic anisotropy, which does not allow for spin-flip processes responsible for the Kondo effect. As the field gets increased the ground state becomes monotonically split, which translates into the occurrence of a dip in differential conductance around $V_{\mathrm{b}}=0$. The position of edges of this dip is marked by dashed lines, from which one can estimate the value of the tunnel spitting $\Delta$. It is also interesting to note that the dip is more pronounced in the parallel magnetic configuration, see Fig. 1b.

The response of the system to magnetic field $B_{x}$ becomes significantly different when the transverse component of magnetic anisotropy is included, Fig. 1c-f. First of all, for $B_{x}=0$ one observes that the linear conductance achieves the unitary limit of $2 e^{2} / h$ due to the Kondo effect. This is especially noticeable for $E / D=1 / 3,(\mathrm{e})-(\mathrm{f})$, whereas for $E / D=1 / 10,(\mathrm{c})-(\mathrm{d})$, it can be barely seen, but closer analysis proves the formation of the Kondo resonance also in this case, albeit with much smaller Kondo temperature. With increasing $B_{x}$, the degeneracy of the ground state doublet is removed, which leads to the splitting of the resonance and formation of a dip in differential conductance. However, unlike for $E=0$, further growth of $B_{x}$ restores the condition $\Delta=0$, which essentially results in the revival of the Kondo effect. For a molecule under discussion with $S^{t}=5 / 2$ (i.e., at $\varepsilon=-U / 2$ when the OL is occupied by a single electron), the degeneracy of the ground state doublet can be recovered with application of $B_{x}$ twice [1]. What is more, the resonant fields, at which the Kondo effect is brought back, do not occur at the constant interval $2 \sqrt{2 E(D+E)}$, as observed for systems described entirely by the giant-spin Hamiltonian (2) [24-26]. This is due to the fact that the model considered here also allows for charging the molecule with additional electrons occupying the orbital level, contrary to the giant-spin Hamiltonian where the molecule is solely described by the anisotropy constants $D$ and $E$. Finally, we note that although the revivals of the Kondo effect occur in both magnetic configurations, this effect is more pronounced in the antiparallel configuration compared to the parallel one, which is associated with smaller Kondo temperature in the latter configuration.

\section{Conclusion}

We have analyzed the effect of transverse magnetic field on the spin-dependent transport through a largespin molecule strongly coupled to external ferromagnetic leads. We have shown that, when the molecule exhibits finite transverse magnetic anisotropy, the magnetic field gives rise to revivals of the Kondo effect, with the Kondo temperature depending on the magnetic configuration of the device.

\section{Acknowledgments}

The use of the SPINLAB computational facility is kindly acknowledged. This work was supported by the Polish Ministry of Science and Higher Education through a research project in years 2010-2013. I.W. was supported by the National Science Center in Poland through the Project No. DEC-2013/10/E/ST3/00213.

\section{References}

[1] D. Gatteschi, R. Sessoli, J. Villain, Molecular Nanomagnets, Oxford University Press, New York 2006.

[2] M. Ternes, A.J. Heinrich, W.-D. Schneider, J. Phys. Condens. Matter 21, 053001 (2009).

[3] M. Mannini, F. Pineider, P. Sainctavit, C. Danieli, E. Otero, C. Sciancalepore, A.M. Talarico, M.A. Arrio, A. Cornia, D. Gatteschi, R. Sessoli, Nature Mater. 8, 194 (2009).

[4] R. Sessoli, D. Gatteschi, A. Caneschi, M.A. Novak, Nature 365, 141 (1993).

[5] L. Thomas, F. Lionti, R. Ballou, D. Gatteschi, R. Sessoli, B. Barbara, Nature 383, 145 (1996).

[6] M. Mannini, F. Pineider, C. Danieli, F. Totti, L. Sorace, Ph. Sainctavit, M.-A. Arrio, E. Otero, L. Joly, J.C. Cezar, A. Cornia, R. Sessoli, Nature 468, 417 (2010).

[7] M. Misiorny, J. Barnaś, Phys. Rev. Lett. 111 , 046603 (2013).

[8] A.C. Hewson, The Kondo Problem to Heavy Fermions, Cambridge University Press, Cambridge 1997.

[9] A.F. Otte, M. Ternes, K. von Bergmann, S. Loth, H. Brune, C.P. Lutz, C.F. Hirjibehedin, A.J. Heinrich, Nature Phys. 4, 847 (2008).

[10] J.J. Parks, A.R. Champagne, T.A. Costi, W.W. Shum, A.N. Pasupathy, E. Neuscamman, S. Flores-Torres, P.S. Cornaglia, A.A. Aligia, C.A. Balseiro, G.K.-L. Chan, H.D. Abruña, D.C. Ralph, Science 328, 1370 (2010).

[11] M. Misiorny, I. Weymann, J. Barnaś, Phys. Rev. Lett. 106, 126602 (2011).

[12] M. Misiorny, I. Weymann, J. Barnaś, Phys. Rev. B 84, 035445 (2011).

[13] C. Romeike, M.R. Wegewijs, W. Hofstetter, H. Schoeller, Phys. Rev. Lett. 96, 196601 (2006).

[14] M. Misiorny, I. Weymann, Phys. Rev. B 90, 235409 (2014).

[15] H. Bruus, K. Flensberg, Many-Body Quantum Theory in Condensed Matter Physics, Oxford Graduate Texts, Oxford University Press, Oxford 2004.

[16] S. Csonka, I. Weymann, G. Zarand, Nanoscale 4, 3635 (2012).

[17] K.G. Wilson, Rev. Mod. Phys. 47, 773 (1975).

[18] R. Bulla, T.A. Costi, T. Pruschke, Rev. Mod. Phys. 80, 395 (2008). 
[19] A. Weichselbaum, J. von Delft, Phys. Rev. Lett. 99, 76402 (2007).

[20] Ö. Legeza, C.P. Moca, A.I. Tóth, I. Weymann, G. Zaránd, arXiv:0809.3143v1, 2008, the code is available at Flexible DM-NRG.

[21] J. Martinek, Y. Utsumi, H. Imamura, J. Barnaś, S. Maekawa, J. König, G. Schön, Phys. Rev. Lett. 91, 127203 (2003).
[22] M. Gaass, A.K. Hüttel, K. Kang, I. Weymann, J. von Delft, Ch. Strunk, Phys. Rev. Lett. 107, 176808 (2011).

[23] I. Weymann, Phys. Rev. B 83, 113306 (2011).

[24] W. Wernsdorfer, R. Sessoli, Science 284, 133 (1999).

[25] A. Garg, Europhys. Lett. 22, 205 (1993).

[26] P. Bruno, Phys. Rev. Lett. 96, 117208 (2006). 